\title{
Micosis superficiales en la ciudad de Valparaíso, Chile. Período 2007-2009
}

\author{
Rodrigo Cruz Ch., Eliette Ponce E., Leslie Calderón R., \\ Natalia Delgado V., Peggy Vieille O. y Eduardo Piontelli L.
}

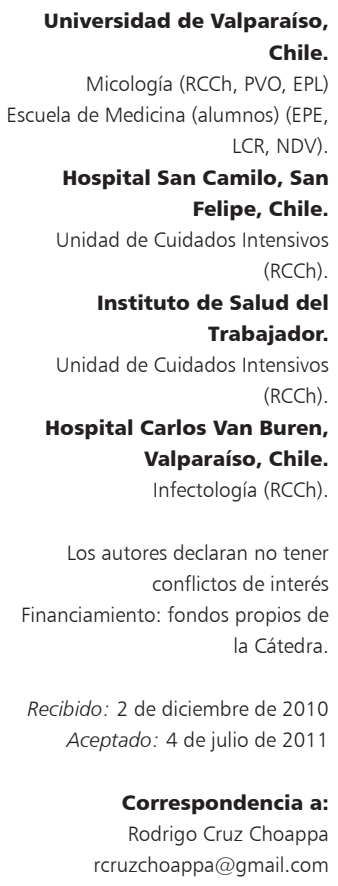

Universidad de Valparaíso

Chile.

Micología (RCCh, PVO, EPL)

Escuela de Medicina (alumnos) (EPE

LCR, NDV).

Hospital San Camilo, San

Felipe, Chile.

Unidad de Cuidados Intensivos

(RCCh).

Instituto de Salud del

Trabajador.

Unidad de Cuidados Intensivos

(RCCh).

Hospital Carlos Van Buren,

Valparaíso, Chile.

Infectología (RCCh).

Los autores declaran no tener conflictos de interés

Financiamiento: fondos propios de la Cátedra.

Recibido: 2 de diciembre de 2010

Aceptado: 4 de julio de 2011

Correspondencia a:

Rodrigo Cruz Choappa rcruzchoappa@gmail.com

\section{Superficial mycoses in the city of Valparaiso, Chile. Period 2007-2009}

Introduction: Superficial mycoses are infections of the keratinous tissue caused by dermatophytes, yeasts and environmental fungi. Objectives: To identify the species of dermatophytes, yeasts and environmental fungi that cause superficial mycosis and analyze their differences by sex, age and location of the lesion, in patients from the region of Valparaiso, during 2007-2009. Materials and Methods: 1004 patients diagnosed with superficial mycoses were studied. All ages and both genera were included. The study was done using direct microscopic examination with $20 \% \mathrm{KOH}$ and culture of the lesions. Fungi identification was mainly morphophysiological. Results: Of the 1004 patients studied, 609 were women and $87.7 \%$ was 15 years old or younger. Toenail onychomycosis was the most frequent lesion $(58.1 \%)$, followed by tinea pedis and interdigitalis $(16.3 \%)$. In patients younger than 8 years old, tinea capitis by Microsporum canis was the most common mycoses. Among dermatophytes, Trichophyton rubrum (78.9\%) predominated in most locations, followed by Trichophyton mentagrophytes (14.9\%) and $M$. canis (5.4\%). Candida spp. (95.4\%) prevailed among the yeasts causing onychomycosis. There were 27 cases of toenail onychomycosis caused by environmental fungi, mainly Fusarium. Conclusions: Our results are consistent with national and international data, where T. rubrum is the predominant agent of dermatomycoses. We report the emergency of agents not described in Chile or Latin America in these infections.

Key words: Superficial mycoses, dermatophytoses, onychomycosis.

Palabras clave: Micosis superficial, dermatofitosis, onicomicosis.

\section{Introducción}

L as micosis superficiales o dermatomicosis son infecciones causadas por hongos en tejidos queratínicos como piel, cuero cabelludo y uñas ${ }^{1,2}$. Las dermatofitosis son causadas por hongos filamentosos que en su estado anamórfico pertenecen a los géneros Trichophyton, Microsporum y Epidermophyton ${ }^{3-5}$; sin embargo, se han descrito estados sexuales de varias especies, incluidas todas en el género Arthrodema, aunque desde el punto de vista clínico se siguen usando las denominaciones anamórficas 5 .

Resulta útil clasificar a los dermatofitos según el medio donde se desarrollan naturalmente; si se encuentran en el suelo se denominan geofílicos o telúricos (Microsporum gypseum), en animales se denominan zoofílicos (Microsporum canis, Trichophyton mentagrophytes var mentagrophytes) y antropofílicos si se desarrollan en el hombre (Trichophyton rubrum, Epidermophyton floccosum) . $^{5}$

Se han realizado estudios de las especies causantes de micosis superficiales en prácticamente todo el mundo, los cuales han mostrado que éstas varían según las áreas geográficas, factores socioeconómicos, climáticos y migraciones poblacionales ${ }^{5,6}$.

Las especies de dermatofitos más frecuentes en nuestro país son T. rubrum, T. mentagrophytes y M. canis $^{1,2,7-9}$. En la Región de Valparaíso, Piontelli y cols ${ }^{7}$, realizaron un estudio sobre las micosis superficiales durante el período 1984-1989, donde predominó T. rubrum en la mayoría de las lesiones del cuerpo y M.canis en tinea capitis. Luego de ese estudio no existen otras referencias que indiquen alguna variación en los porcentajes de las especies dominantes o la aparición de nuevas especies en esta región.

Las levaduras siguen en frecuencia a los dermatofitos, siendo las pertenecientes al género Candida las más relevantes, especialmente C. albicans que puede provocar tanto infecciones de piel como de uñas ${ }^{10}$. Géneros como Trichosporon spp y Rodhotorula spp pueden provocar onicomicosis ${ }^{10,11}$ y las especies del complex Malassezia se describen asociadas principalmente a pitiriasis versicolor $^{12}$

Ocasionalmente, hongos ambientales, tales como Fusarium spp, Scopulariopsis spp, Aspergillus spp y Acremonium spp, entre otros géneros, pueden causar in- 
fecciones o sobreinfecciones oportunistas, especialmente en uñas ${ }^{13}$.

La importancia de reconocer las distintas especies de dermatofitos, levaduras y hongos ambientales en el diagnóstico de una micosis superficial es útil no solamente en la decisión terapéutica, sino también en la prevención de re-infecciones y en la determinación de cambios en las frecuencias de las distintas especies a través del tiempo ${ }^{14}$.

El objetivo de este estudio fue conocer las especies de dermatofitos, levaduras y hongos ambientales que causan dermatomicosis, además de analizar las diferencias según sexo, edad y localización de estas infecciones en pacientes de la Región de Valparaíso.

\section{Materiales y Métodos}

Estudio de tipo descriptivo, prospectivo. Se evaluaron 1.004 pacientes de todas las edades y de ambos sexos, consultantes en la Región de Valparaíso, con diagnóstico de micosis superficial durante el período 2007-2009.

Criterios de inclusión: Pacientes con lesión sugerente de dermatomicosis (evaluada por dermatólogo tratante), más examen microscópico directo con estructuras fúngicas y/o cultivo con desarrollo de hongos filamentosos o levaduras a partir de la lesión. Si el resultado del examen microscópico directo (micelio o levaduras) coincidió con el cultivo (dermatofitos, Candida spp u otra levadura), se asumió como agente etiológico a la especie desarrollada en este último. El diagnóstico de onicomicosis de pie por hongos ambientales se realizó con tres exámenes microscópicos directos con elementos fúngicos más tres cultivos a $37^{\circ} \mathrm{C}$ con desarrollo del mismo moho, tomados en tiempos distintos.

Diagnóstico micológico: Se realizó en la Cátedra de Micología de la Escuela de Medicina de la Universidad de Valparaíso. Las muestras de las lesiones fueron obtenidas por personal entrenado y depositadas en placas de Petri estériles para evitar contaminación. El examen microscópico directo se realizó con $\mathrm{KOH}$ al 20\% para detectar la presencia de elementos fúngicos mediante microscopia óptica. El cultivo se realizó en agar Sabouraud glucosado y agar Lactrimel, ambos adicionados con cloranfenicol
$(125 \mu \mathrm{g} / \mathrm{ml})$ e incubadas a 27 y $37^{\circ} \mathrm{C}$ durante tres semanas. La identificación de los hongos filamentosos fue en base a su morfo-fisiología. En el caso de las levaduras, el estudio fue principalmente a nivel de género, siendo la identificación y sensibilidad de éstas motivo de otra investigación en curso. En los casos específicos de pitiriasis versicolor, se empleó la técnica de muestreo de cinta adhesiva, tinción con safranina y observación microscópica directa, lo que permitió el diagnóstico a nivel de género del complex Malassezia.

Análisis estadístico: Se realizó con $\chi^{2}$ de Pearson para establecer la significancia de las variables cualitativas entre sí y ANOVA de una vía para analizar la distribución de variables por edad.

Se consignaron en ficha ad-hoc, las variables edad, sexo, ubicación de la lesión, resultado del examen microscópico directo y del cultivo, para cada paciente.

\section{Resultados}

De los 1.004 pacientes incluidos en el estudio, 609 $(60,6 \%)$ correspondieron al sexo femenino y $880(87,6 \%)$ tenían sobre 15 años de edad. Las dermatomicosis fueron más frecuentes en el grupo entre 36 y 60 años (Figura 1), con un promedio de 41,5 años.

La presentación clínica más frecuente entre las micosis superficiales fue la onicomicosis de pie $(58,1 \%)$, seguida de tinea plantar e interdigital (16,3\%). La tinea corporis, tinea capitis y otras localizaciones fueron menos frecuentes (Tabla 1).

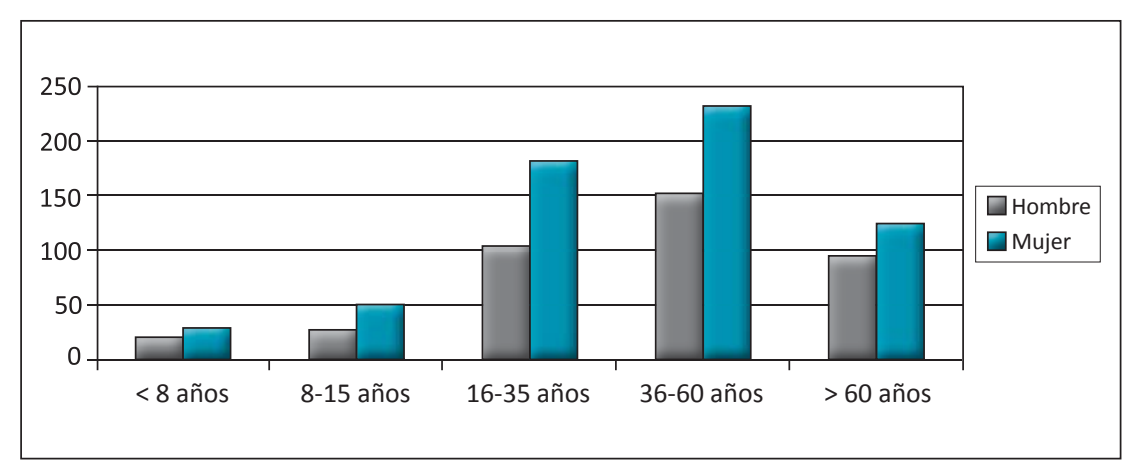

Figura 1. Distribución de las micosis por edad y sexo.

\begin{tabular}{|c|c|c|c|c|c|c|c|c|}
\hline Sexo & & $\begin{array}{l}\text { Cuero } \\
\text { cabelludo }\end{array}$ & $\begin{array}{c}\text { Palmar- } \\
\text { interdigital }\end{array}$ & $\begin{array}{c}\text { Plantar- } \\
\text { interdigital }\end{array}$ & Uña de la mano & Uña del pie & Resto de la piel & Total \\
\hline Femenino & n (\%) & $14(2,2 \%)$ & $58(9,5 \%)$ & $96(15,7 \%)$ & $47(7,7 \%)$ & $362(59,4 \%)$ & $32(5,2 \%)$ & $609(100 \%)$ \\
\hline Masculino & n (\%) & $10(2,5 \%)$ & $35(8,8 \%)$ & $68(17,2 \%)$ & $33(8,3 \%)$ & $221 \quad(55,9 \%)$ & $28(7 \%)$ & $395(100 \%)$ \\
\hline
\end{tabular}


No se observó una diferencia estadísticamente significativa entre sexos y la localización de las micosis superficiales (p: 0,784). Sólo la onicomicosis de manos provocada por levaduras se presentó con mayor frecuencia en mujeres ( $p$ : 0,046); sin embargo, se observaron diferencias en las localizaciones de las lesiones por rango de edad, donde tinea capitis se presentó principalmente en niños bajo 8 años de edad ( $84 \%$ de los casos), tinea corporis en niños entre 8 y 15 años y onicomicosis de manos en adultos sobre 61 años de edad (33,8\%), (p: 0,003).

Del total de micosis superficiales, 133 casos $(13,2 \%)$ fueron diagnosticados sólo con examen microscópico directo, quedando con diagnóstico de dermatomicosis por hongo filamentoso o levaduras, $155(15,4 \%)$ sólo con cultivo y $716(71,3 \%)$ con examen microscópico directo y cultivo (en estos dos últimos casos realizándose el diagnóstico etiológico). Por lo tanto, el rendimiento fue similar entre el examen microscópico directo y el cultivo en el diagnóstico de una micosis superficial (84,6 y 86,7\%, respectivamente).

En 871 pacientes hubo desarrollo fúngico en el cultivo, de los cuales 550 fueron por hongos filamentosos (523 dermatofitos y 27 hongos ambientales) y 321 por levaduras.

Entre los dermatofitos se observó una mayor frecuencia de T. rubrum, seguido por T. mentagrophytes y M. canis (Tabla 2). Trichophyton rubrum predominó en la mayoría de las lesiones; en cambio $M$. canis fue la especie más frecuente en cuero cabelludo de niños bajo 8 años de edad. Destaca el escaso aislamiento de Epidermophyton floccosum (sólo dos casos), además del diagnóstico de un caso de tinea capitis por Microsporum audouinii y un caso de tinea corporis por Microsporum praecox (Figura 2).

Entre los hongos levaduriformes, se observó de mayor a menor frecuencia Candida spp, Trichosporon spp, Rhodotorula spp y un caso de Cryptococcus laurentii (todos agentes de onicomicosis) (Tabla 3), además de 24 casos de pitiriasis versicolor por Malassezia complex, en cuyo caso $95,8 \%$ de los pacientes presentaban las lesiones en el tronco.

El principal agente en onicomicosis de pie fue $T$. rubrum (45\%), seguido de Candida spp (30\%) y $T$. mentagrophytes $(9,6 \%)$. Además se observaron 27 casos de hongos filamentosos ambientales como causa de onicomicosis de pies, destacando por frecuencia Fusarium solani, Fusarium oxysporum y Scopulariopsis brevicaulis (Tabla 2).

En onicomicosis de mano predominaron las levaduras (95\% por Candida spp), especialmente en mujeres; en cambio, en hombres predominaron los dermatofitos (Tabla 4).

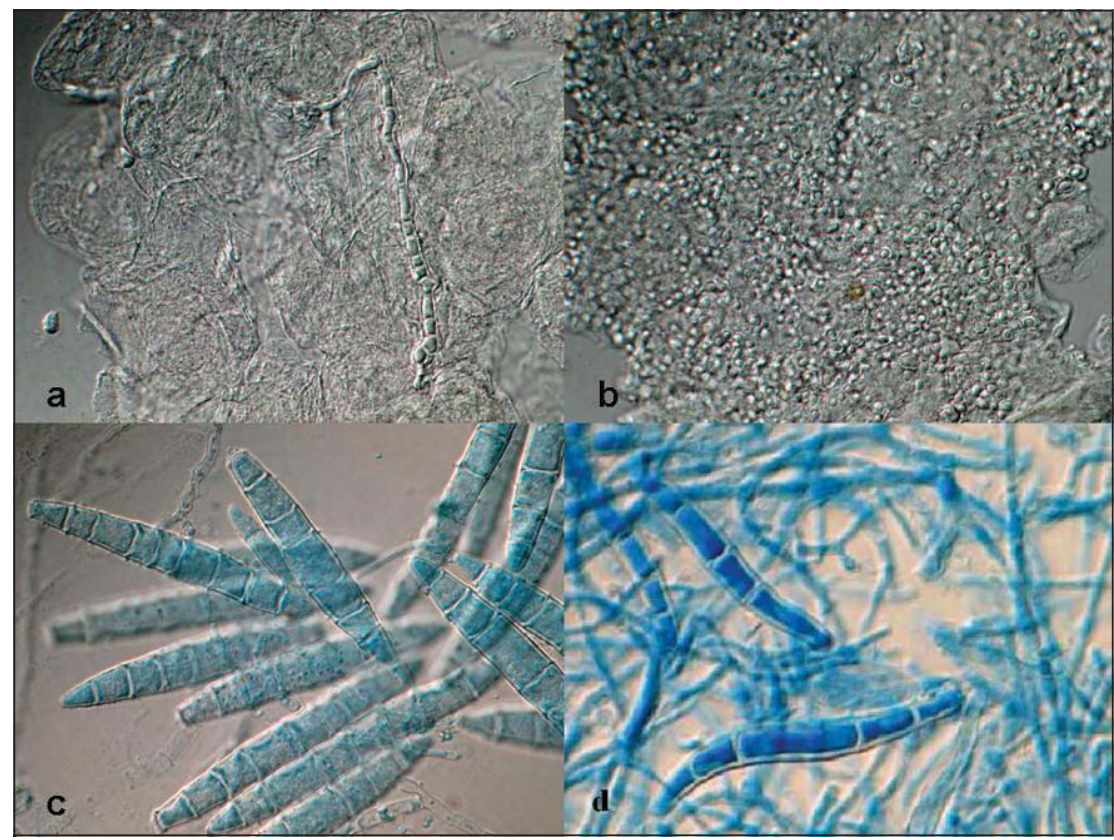

Figura 2. Microscopia DIC. Aumento 1000X: a. Micelio septado de Trichophyton rubrum; b. Microconidios en mosaico de Microsporum canis; c. Macroconidios maduros de Microsporum praecox, alargados, de pared delgada y finamente rugosa, sin microconidios; d. Macroconidios de Microsporum audouinii, con forma de huso (irregular), de pared engrosada, levemente rugosos en las puntas, sin microconidios.

\begin{tabular}{|c|c|c|}
\hline \multicolumn{3}{|l|}{ Dermatofitos en distintas lesiones } \\
\hline Especie fúngica & $n$ & (\%) \\
\hline Trichophyton rubrum & 413 & $(78,9)$ \\
\hline Trichophyton mentagrophytes & 78 & $(14,9)$ \\
\hline Microsporum canis & 28 & $(5,4)$ \\
\hline Epidermophyton floccosum & 2 & $(0,4)$ \\
\hline Microsporum audouinii & 1 & $(0,2)$ \\
\hline Microsporum praecox & 1 & $(0,2)$ \\
\hline \multicolumn{3}{|c|}{$\begin{array}{l}\text { Hongos filamentosos ambientales en } \\
\text { onicomicosis de pies }\end{array}$} \\
\hline Fusarium solani & 10 & $(37,0)$ \\
\hline Fusarium oxysporum & 5 & $(18,5)$ \\
\hline Scopulariopsis brevicaulis & 5 & $(18,5)$ \\
\hline Acremonium strictum & 3 & $(11,1)$ \\
\hline Acremonium kiliense & 1 & $(3,70)$ \\
\hline Aspergillus fumigatus & 1 & $(3,70)$ \\
\hline Aspergillus niger & 1 & $(3,70)$ \\
\hline Alternaria alternata & 1 & $(3,70)$ \\
\hline Total hongos filamentosos & 550( & 100) \\
\hline
\end{tabular}


Tabla 3. Géneros de hongos levaduriformes aislados en onicomicosis de manos y pies

\begin{tabular}{lrc|}
\hline Género & $\mathrm{n}$ & $(\%)$ \\
Candida spp & 306 & $(95,3 \%)$ \\
\hline Trichosporon spp & 11 & $(3,4 \%)$ \\
Rhodotorula spp & 3 & $(0,9 \%)$ \\
Cryptococcus laurentii & 1 & $(0,3 \%)$ \\
Total & 321 & $(100 \%)$ \\
\hline
\end{tabular}

Tabla 4. Hongos filamentosos y levaduriformes aislados en onicomicosis de manos y pies según sexo

\begin{tabular}{|lccc|}
\hline Sexo & $\begin{array}{c}\text { Hongos } \\
\text { filamentosos }\end{array}$ & $\begin{array}{c}\text { Hongos } \\
\text { levaduriformes }\end{array}$ & Total \\
Onicomicosis de & mano & & \\
Femenino & 15 & 33 & 48 \\
Masculino & 15 & 11 & 26 \\
Total & 30 & 44 & 74 \\
Onicomicosis de pie & & & \\
Femenino & 201 & 110 & 311 \\
Masculino & 142 & 77 & 219 \\
Total & 343 & 187 & 530 \\
\hline
\end{tabular}

\section{Discusión}

Las micosis cutáneas constituyen un grupo heterogéneo de infecciones frecuentes en la práctica clínica diaria, donde cada país y región tiene una micota característica, con prevalencia de las distintas especies y formas clínicas que presentan variaciones entre los distintos lugares estudiados ${ }^{7,14}$.

En esta casuística, el diagnóstico de las micosis superficiales aumentó después de los 16 años de edad y disminuyó luego de los 61 años, coincidiendo con estudios realizados tanto en Chile como en otros países, donde se demuestra que el mayor porcentaje de las dermatomicosis se diagnostica en la edad media de la vida ${ }^{2,15,16}$. No se observaron diferencias significativas en cuanto al tipo de lesión y sexo. En ambos géneros hubo una mayor frecuencia de micosis en uñas, planta de pie e interdigital. Sólo en el subgrupo de onicomicosis de manos provocada por levaduras se presentó una mayor frecuencia en mujeres. En un estudio publicado anteriormente en Chile ${ }^{16}$, se describe una mayor frecuencia de onicomicosis en manos de mujeres y de tinea cruris en hombres.

La buena correlación encontrada entre el examen microscópico directo con $\mathrm{KOH}$ al $20 \%$ y el cultivo demuestra que este tipo de exámenes son de mucha utilidad, se potencian y facilitan el diagnóstico ${ }^{17}$. Los resultados negativos en el cultivo se podrían explicar por el uso de medicamentos antimicóticos o por una escasa cantidad de muestra obtenida.

La frecuencia encontrada de las distintas especies concuerda, en general, con la bibliografía nacional y mundial, donde el principal agente causante de micosis superficial fue T. rubrum, seguido de Candida spp., $T$. mentagrophytes y $M$. canis $^{7,16,18,19}$.

La especie dominante en personas sobre los 8 años, en todas las localizaciones y ambos sexos fue T. rubrum. La tinea capitis fue más frecuente en los pre-púberes bajo 8 años de edad y $M$. canis el agente principal, con casos aislados en los demás grupos etáreos.

Hubo un caso de tinea capitis por M. audouinii, en un niño de 8 años que no había salido del país hasta el momento del diagnóstico. Este hongo es considerado antropofílico, habiendo sido aislados en Norteamérica y en Europa; sin embargo, su mayor presencia es en África en niños pre-púberes de escasos recursos, en quienes provoca áreas de alopecia que se expanden lentamente, con mínima o nula inflamación ${ }^{20}$. Actualmente, se reconocen dos variedad de $M$. audouinii (langeronii y rivalier), la primera originaria de África y más patógena en cobayos, cuyas colonias son vellosas, pulverulentas, con surcos radiales y un color asalmonado, mientras que la segunda con colonias de color blanco grisáceo, de crecimiento rápido, aspecto plegado y más vellosas, especialmente en presencia de extracto de levadura, siendo aislada en Norteamérica. Microscópicamente, pueden observarse hifas pectinadas, con clamidosporas terminales o intercalares y raramente macroconidios o microconidios. Microsporium audouinii es a veces difícil de diferenciar de $M$. canis; para ello se puede realizar un cultivo en granos de arroz, donde $M$. canis crece abundantemente y $M$. audouini no crece o lo hace muy pobremente ${ }^{21,22}$. En nuestro caso, tanto la visión macroscópica, cultivo en arroz y la visión microscópica fueron coincidentes con la especie nombrada.

Por primera vez en Chile ${ }^{23}$, se describe un caso de tinea corporis por $M$. praecox en una mujer de 18 años, quien no había salido del país. Este agente es una especie poco común que vive en el suelo y en el pelaje de los animales, en especial el caballo y sus ambientes circundantes. La literatura científica limitaba hasta ahora su distribución a Europa, en especial Francia y Bélgica ${ }^{24,25}$ y raramente en E.U.A. ${ }^{26}$.

Epidermophyton floccosum fue aislado sólo en dos casos (tinea cruris y tinea ungium), demostrando que su presencia ha disminuido con el tiempo, tanto en Chile como en otros países, siendo desplazado principalmente por T. rubrum $2,27,28$.

Los hongos levaduriformes (principalmente Candida spp) produjeron tanto onicomicosis de pies como de 
manos; sin embargo, fueron significativamente más frecuentes en uñas de manos de mujeres que de hombres. Algunos trabajos no muestran diferencias significativas por género, tanto en hongos levaduriformes como en dermatofitos ${ }^{29,30}$, mientras en otros la onicomicosis es significativamente más frecuente en hombres ${ }^{31}$. Esto se podría explicar por la población incluida en cada estudio, sus áreas geográficas, condiciones laborales que las expone a condiciones de humedad, traumatismos que favorecen el crecimiento de hongos o por un sesgo debido a que las mujeres consultan más por un interés estético.

Las especies de hongos ambientales son de baja frecuencia y pueden producir onicomicosis en pies. $\mathrm{Su}$ tratamiento puede ser más dificultoso debido a la resistencia que presentan a varios de los antimicoticos usados. En nuestra serie destacaron por su frecuencia F. solani, F. oxysporum, y S. brevicaulis, resultado que coincide con investigaciones anteriores, donde Fusarium spp predominan sobre otras especies ${ }^{13,32,33}$.

Si bien nuestro trabajo incluyó sólo a pacientes de la Región de Valparaíso, es un avance en la identificación de los principales agentes de dermatomicosis y en demostrar la aparición de hongos no registrados anteriormente en Chile y Latinoamérica. Esto ayuda en la elección de un tratamiento adecuado y en las medidas de prevención de estas infecciones.

Agradecimientos. A los profesores Eduardo Piontelli y M. Alicia Toro de la Universidad de Valparaíso, por sus infinitas enseñanzas y apoyos.

\section{Resumen}

Introducción: Las micosis superficiales son infecciones causadas por dermatofitos, levaduras y hongos ambientales en tejidos queratínicos. Objetivos: Conocer las especies de dermatofitos, levaduras y hongos ambientales que causan micosis superficiales, analizar sus diferencias según sexo, edad y localización en pacientes de la Región de Valparaíso durante el período 2007-2009. Materiales y Métodos: Se estudiaron 1.004 pacientes de todas las edades, con diagnóstico de micosis superficiales. Se realizó examen microscópico directo con $\mathrm{KOH}$ al 20\% y cultivos de las lesiones. La identificación de los hongos fue principalmente con morfo-fisiología. Resultados: De 1.004 pacientes evaluados, 609 fueron mujeres y $87,7 \%$ tenían sobre 15 años de edad. La onicomicosis de pie fue la lesión más frecuente $(58,1 \%)$, seguida de tinea plantar e interdigital (16,3\%). Bajo 8 años de edad, tinea capitis por Microsporum canis fue la lesión más frecuente. Entre los dermatofitos, Trichophyton rubrum (78,9\%) predominó en la mayoría de las localizaciones, seguido por Trichophyton mentagrophytes $(14,9 \%)$ y $M$. canis $(5,4 \%)$. Entre las levaduras predominó Candida spp $(95,4 \%)$ como causa de onicomicosis. Hubo 27 casos de onicomicosis de pies por hongos filamentosos ambientales, destacando por frecuencia el género Fusarium. Conclusiones: Los datos presentados coinciden en general con los trabajos nacionales e internacionales, donde T. rubrum predomina en la mayoría de las dermatomicosis. Destaca la aparición de agentes no descritos en Chile ni en Latinoamérica en este tipo de infecciones.

\section{Referencias}

1.- Arenas R. Dermatofitosis. Arenas R, editor. Micología Médica Ilustrada, Ia ed. Ciudad de México: Editorial McGraw-Hill; 2004, p. 61-94.

2.- Alarcón R, Pérez M, Rodríguez M, Herlizt H, Solís F. Agentes etiológicos de dermatomicosis aislados en pacientes de la ciudad de Concepción y comunas circunvecinas. 2006. Rev Chil Dermatol 2008; 24: 109-15.

3.- Del Palacio A, Garaul M, Cuétara M. Tratamiento actual de las dermatofitosis. Rev Iberoam Micol 2002; 19: 68-71.

4.- Segundo C, Martínez A, Arenas R, Fernández R, Cervantes R. Dermatomicosis por Microsporum canis en humanos y animales. Rev Iberoam Micol 2004; 21 (1): 39-41.

5.- Velasco M, García-Melgares L, Gimeno E, Roche E, Vilata J J. Dermatofitosis. Vilata J J, editor. Micosis Cutaneas, $\mathrm{I}^{\mathrm{a}}$ ed. Madrid: Editorial Médica Panamericana 2006; p. 49-72.

6.- Van breuseghem, R, De Vroey C H. Geographic distribution of dermatophytes. Int J Dermatol 1970; 9: 102-9.

7.- Piontelli E, Toro M, Casanova D, Jara D. Micosis superficiales en pacientes de servicios dermatológicos de la V Región: Estudios de prevalencia en el período 1984-1989. Bol Micol 1991; 6: 63-8.

8.- Zaror L, Moreno M, Vega K, Hering M, Frick P. Agentes de onicomicosis en manos y pies en Valdivia (Chile). Bol Micol 1995; 10: 53-8.

9.- Díaz M, Fich F, Salamanca L. Agentes etiológicos de micosis superficiales en una área de Santiago-Chile (1977-1987). Bol Micol 1990; 5: 5-8.

10.- Jayatilake J A, Tilakaratne W M, Panagoda G J. Candidal onychomycosis: a mini-review. Mycopathologia 2009; 168 (4): 165-73.

11.- Valdivia-Blondet L, Escalante-Jibaja E, Domínguez N, Correa C, Quintero J, Córdova A, et al. Estudio etiológico de la onicomicosis pedis en personal militar en formación. Dermatol Peru 2000; 10 (2): 89-93.
12.- Crespo-Erchiga V, Florencio V. Malassezia yeasts and pityriasis versicolor. Curr Opin Infect Dis 2006; 19: 139-47.

13.- Cavallera E, Asbati M. Onicomicosis por hongos filamentosos no dermatofitos. Dermatol Venez 2006; 44: 4-10.

14.- Larrondo R, González A, Hernández L. Micosis superficiales. Dermatofitosis. Rev Cubana Med Gen Integr 2001; 17: 59-64.

15.- Runco R, Salim R, Silva J. Dermatomicosis en niños: Cinco años de estudios en TucumánArgentina. Bol Micol 2000; 15: 57-63.

16.- Mazón A, Salvo S, Vives R, Valcayo A, Sabalza M. Estudio etiológico y epidemiológico de las dermatofitosis en Navarra (España). Rev Iberoam Micol 1997; 14: 65-8.

17.- Merino D, Honeyman J, Larrondo J, Gosch M, De la Parra R, Zapata S. Diferencias por género en el diagnóstico de micosis superficiales: Análisis de 30.590 pacientes. Rev Chil Dermatol 2009; 25 (1): 57-61.

18.- Miller M A, Hodgson Y. Sensitivity and 
specificity of potassium hydroxide smears of skin scrapings for the diagnosis of tinea pedis. Arch Dermatol 1993; 129 (4): 510-1.

19.- Mugge U, Nenoff P. Causative agents of onichomycosis a retrospective study. J Dtsch Dermatol Ges 2006; 4: 218-27.

20.- Purim K S, Bordignon G P, Queiroz-Telles F. Fungal infection of the feet in soccer players and non-athlete individuals. Rev Iberoam Micol 2005; 22: 34-8.

21.- Rebollo N, López-Barcenas A P, Arenas R. Tiña de la cabeza. Actas Dermosifiliogr 2008; 99 : 91-100.

22.- Pereiro-Miguens M, Pereiro M. Dermatofitosis y sus agentes etiológicos. Torres Rodríguez JM, editor. Micología Médica, I ${ }^{a}$ ed. Barcelona: Editorial Masson; 1993, p 103-129.

23.- Escutia B, Febrer I, Pemán J, Oliver V, Sánchez-Carazo J L. Tinea capitis por Microsporum audouinii. Rev Iberoam Micol 2001; 18: 88-90.

24.- Piontelli E, Vivar V. Casos Clínicos:
Microsporum praecox y Acremonium strictum. Nuevos agentes de micosis cutáneas oportunistas en la zona central de Chile. Bol Micol 2007; 22: 55-63.

25.- DeVroey C, Wuytack-Raes C, Fossoul F. Isolation of saprophytic Microsporum praecox Rivalier from sites associated with horses. Sabouraudia 1983; 21: 255-7.

26.- Aertgeerts P, DeVroey C. Isolement de Microsporum praecox Rivalier en Belgique. Med Mycol 1981; 10: 17-20.

27.- Weitzman I, Mc Millen S. Isolation in the United States of a culture resembling Microporum praecox. Mycopathologia 1980; 70: 181-6.

28.- Rubio M C, Rezusta1 A, Gil Tomás J, Ruescal R B. Perspectiva micológica de los dermatofitos en el ser humano. Rev Iberoam Micol 1999; 16: 16-22.

29.- Padilla A, Sampedro A, Sampedro P, Delgado V. Estudio clínico y epidemiológico de las dermatofitosis en una zona básica de salud de
Jaén (España). Rev Iberoam Micol 2002; 19 : 36-39.

30.- Medina J, Bejar V, Cortez F, Betanzos A. Superficial fungal infections: clinical and epidemiological study in adolescents from marginal districts of Lima and Callao, Peru. J Infect Dev Ctries 2009; 3(4): 313-7.

31.- Walling H W. Subclinical onychomycosis is associated with tinea pedis. Br J Dermatol 2009; 161(4): 746-9.

32.- Perea S, Ramos M J, Garau M, González A, Noriega A, Del Palacio A. Prevalence and risk factors of tinea unguium and tinea pedis in the general population in Spain. J Clin Microbiol 2000; 38 (9): 3226-30.

33.- Escobar M L, Carmona-Fonseca J. Onicomicosis por hongos ambientales no dermatofiticos. Rev Iberoam Micol 2003; 20 : 6-10.

34.- Albrecht M C, Fraenza L B, Tramonda G. Onicomicosis por Acremonium kiliense. Rev Argent Dermatol 2007; 88: 40-4. 